A Clinical Translation of the Research Article Titled "Antisocial Behavioral Syndromes and Additional Psychiatric Comorbidity in Posttraumatic Stress

Disorder among US Adults: Results from Wave 2 of the National Epidemiologic Survey on Alcohol and Related Conditions"

Colleen Corte, $\mathrm{PhD}, \mathrm{RN}$ 


\section{STUDY PURPOSE:}

The purpose of the study conducted by Goldstein and colleagues was to compare the lifetime prevalence of major mental disorders and personality disorders in three groups of adults who had posttraumatic stress disorder (PTSD): 1) those who met diagnostic criteria for Antisocial Personality Disorder (ASPD), 2) those who had antisocial behavior in adulthood but did not meet the full diagnostic criteria for ASPD because of the absence of conduct disorders in childhood (Adult Antisocial Behavioral Syndrome), and 3) those who did not have antisocial behavior. Adult antisocial behavioral syndrome (AABS) is not a recognized psychiatric disorder in the DSM-IV. However, given the high symptom severity and rates of other psychiatric disorders associated with AABS, the authors wanted to determine whether adults with PTSD and AABS had similar numbers and types of psychiatric disorders compared to adults with PTSD and ASPD, or whether they were more similar to adults with PTSD who had no antisocial behavior. The "other" psychiatric disorders measured included mood disorders (major depressive disorder, dysthymia, bipolar I disorder, and bipolar II disorder), other anxiety disorders (panic disorder, social phobia, specific phobia, and generalized anxiety disorder), substance use disorders (alcohol abuse or dependence, drug abuse or dependence, nicotine dependence), attention deficit hyperactivity disorder (ADHD), and other personality disorders (paranoid, schizoid, schizotypal, histrionic, narcissistic, and borderline).

\section{BACKGROUND:}

Antisocial personality disorders are much more prevalent in persons with PTSD than they are in the general population. This is intriguing given that ASPD is more common in men and PTSD is more common in women. Some researchers argue, however, that because the diagnostic criteria for ASPD (and for childhood conduct disorders which antedate ASPD) focus on overtly 
aggressive behaviors which are more characteristic of males, rather than relational forms of aggressive behavior which are more characteristic of females, the higher prevalence of ASPD (and childhood conduct disorders) in males may be due to an artifact of the diagnostic criteria rather than due to a true male-female difference in prevalence.

Although ASPD and PTSD are both frequently associated with co-occurring psychiatric disorders and chronic medical conditions which often lead to poor treatment outcomes, no studies to date have examined whether ASPD is associated with the severity of clinical presentation in PTSD, including the rates and patterns of psychiatric comorbidity. A diagnosis of ASPD requires both the presence of conduct disorders prior to age 15 and antisocial behavior after age 15 and into adulthood. No DSM diagnosis currently exists for persons with antisocial behavior in adulthood who do not have a diagnosis of conduct disorders prior to age 15, even though the severity of their antisocial behavior and degree of their psychiatric comorbidity are similar to that seen in adults who meet diagnostic criteria for ASPD. The authors of this study argue that classifying adults with AASB simply as "persons without ASPD" may obscure potentially important differences in clinical presentation and treatment outcomes of PTSD because persons with AASB are likely to be quite different than adults without any antisocial behavior.

\section{METHOD:}

This study reports an analysis of existing data (secondary analysis) from a single time point (cross-sectional), wave 2 (the second data collection point which in this case was a 3-year follow-up from the wave 1 or baseline data collection) of the ongoing study, from a large nationally representative sample of non-institutionalized adults aged 18 or older in the US from the National Epidemiologic Survey on Alcohol and Related Conditions (NESARC). A 
"nationally representative" sample of non-institutionalized adults means that all adults 18 years of age and older who were residing in households rather than hospitals or other institutions had an equal chance of participating in the study. Such a sample allows the investigators to determine fairly precise estimates of the prevalence of particular conditions among adults in the US population at large. In fact, randomly selecting a large sample of persons from the population at large yields virtually the same information as what you would find if you included every single person in the US who met the study inclusion criteria, in this case, all persons who were 18 years or older and not living in an institutionalized setting. Of the 34,653 participants, 2,442 had PTSD and were included in the analyses. The investigators were interested in persons with PTSD who differed in terms of their level of antisocial behavior. They categorized the 2,442 persons with PTSD into one of three groups: 177 had ASPD, 629 had AABS, and 1,636 did not have any antisocial behavior syndrome.

The original data were collected via face-to-face interviews using the Alcohol Use Disorder and Associated Disabilities Schedule DSM-IV Version (AUDASIS-IV) developed by the National Institute on Alcohol Abuse and Alcoholism at the National Institutes of Health. This is a structured computerized interview designed to be administered by non-clinicians. The aim of a structured interview is to ensure that each interview consists of exactly the same questions in exactly the same order. The variables (in this case diagnoses or conditions) used in this particular investigation included: presence or absence of 1) a lifetime diagnosis of PTSD, 2) a lifetime diagnosis of ASPD, 3) a lifetime history of AABS (there is no official diagnosis for AABS), 4) a lifetime diagnosis of mood disorders, other anxiety disorders, substance use disorders, and ADHD, and 5) a lifetime diagnosis of paranoid, schizoid, schizotypal, histrionic, narcissistic, and borderline personality disorders. 


\section{RESULTS:}

The findings of this study are presented in terms of comparisons of three groups of participants: those with PTSD and comorbid ASPD, those with PTSD and comorbid AABS, and those with PTSD who had no comorbid antisocial behavioral syndrome. The three groups were compared in terms of sociodemographic characteristics, psychiatric history of family members, and rate and pattern of co-occurring psychiatric disorders in the participant.

Sociodemographic Characteristics: The key findings with respect to sociodemographics are that among persons with PTSD: 1) those who have either ASPD or AABS were more likely to be male and more likely to be younger than persons with PTSD who do not have any antisocial behavioral syndrome; 2) men and women with ASPD or AABS were least likely to identify as Asian or Pacific Islander; 3) men with ASPD were most likely to identify as Hispanic; 4) those with a diagnosis of ASPD were least likely to have attained at least a high school education; and 5) those with AABS were least likely to report ever having had a romantic partner.

Psychiatric History in the Family: Among persons with PTSD, the highest rates of family psychopathology were found in persons who also had ASPD, followed by those who also had AABS. The lowest rates of psychopathology were found in those who did not have any antisocial behavioral syndrome.

Co-Occurring Psychiatric Disorders: Among persons with PTSD, comorbidity rates were very high for all three groups - those with ASPD, those with AABS, and those without any antisocial behavioral syndrome. Most participants met diagnostic criteria for at least one mood disorder, at least one additional anxiety disorder (in addition to PTSD), at least one alcohol or drug use disorder, nicotine dependence, and at least one personality disorder (in addition to 
ASPD). Almost all disorders were associated with having antisocial behavior (either ASPD or AASB). On average, persons with ASPD had 8.2 additional psychiatric disorders including major mental disorders and personality disorders, whereas persons with no antisocial behavioral syndrome had 3.0 additional psychiatric disorders.

After adjusting for the effects of sociodemographic differences (neutralizing the effects of differences in age, sex, race, education), persons with PTSD and ASPD were more 16 times more likely to have histrionic personality disorder, 10 times more likely to have an alcohol or drug use disorder, 6 times more likely to have paranoid or schizoid personality disorder, and 5 times more likely to have bipolar I disorder compared to persons with PTSD who had no antisocial behavioral syndrome. The same pattern was found among persons with PTSD and AABS, but the effects were weaker (ranging from as low as 3 times more likely to as high as 6 times more likely). After further adjusting for the number of additional psychiatric disorders (neutralizing the effects of both sociodemographic differences and differences in the number of additional psychiatric disorders), the patterns of association with specific co-occurring psychiatric disorders were similar though the strength of these associations was somewhat weaker.

The investigators also found some "interactions" between sex and antisocial syndrome for three major mental disorders (major depressive disorder, bipolar II, and generalized anxiety disorder) and one personality disorder (dependent personality disorder). These interactions mean that the rates of these disorders in persons with PTSD varied according to the degree of antisocial behavior, but the patterns of co-occurrence varied differently for men and women. For major depressive disorder: among men with PTSD, the highest rates were found in those with AABS and the lowest rates were found in those with ASPD; but among women with PTSD, the highest 
rates were found in those with no antisocial behavioral syndrome and the lowest rates were found in those with ASPD. For bipolar II disorder: among men with PTSD, the highest rates were found for those with ASPD, and the lowest rates were found in those with AABS; among women with PTSD, the highest rates were also found in those with ASPD, but the lowest rates were found in those without any antisocial behavior syndrome. For generalized anxiety disorder: among men with PTSD, rates were highest in those with AABS and lowest in those with either ASPD or no antisocial behavioral syndrome; among women with PTSD, rates were highest in those with ASPD and lowest in those with no antisocial behavioral syndrome. Finally, for dependent personality disorder: though the rates were highest in both men and women with PTSD and ASPD and lowest in those with PTSD but no antisocial behavioral syndrome, the differences between groups of persons with PTSD (ASPD, AABS, and no antisocial behavioral syndrome) were much more striking among women than among men. These patterns generally persisted regardless of adjustments for sociodemographic characteristics and the number of additional psychiatric disorders.

\section{DISCUSSION:}

This was the first study to investigate the rates and types of co-occurring psychiatric disorders in persons with PTSD who also had ASPD, AABS, or no antisocial behavioral syndrome. Very high rates of additional lifetime psychiatric disorders were found in all groups. Generally, those with ASPD had the highest rates of comorbidity, followed by those with AABS, followed by those with no antisocial behavioral syndrome. One of the most important findings of this study is that persons with AABS had significantly higher rates of co-occurring psychiatric disorders than those with no antisocial behavior which suggests that AABS is clinically very important despite the fact that no official AABS diagnosis currently exists in the DSM. The 
authors suggest that either childhood conduct disorders be eliminated as a requisite criterion for ASPD or a new diagnostic category of AABS be created.

There were interesting and unexpected findings with respect to gender differences. Although a diagnosis of ASPD is most strongly associated with co-occurring psychiatric disorders, men with ASPD (but not men with AABS) had lower odds of having two types of disorders -- major depressive disorder and generalized anxiety disorder. Interestingly, both of these disorders are more common in women. The reviewers call for further study of these unexpected findings.

The authors offered possible explanations for the co-occurrences of specific disorders in persons with antisocial behavioral syndromes and PTSD. Comorbid associations between ASPD and dependent and avoidant personality disorders in persons with PTSD may be related to inadequate levels of social support. Possible reasons for this include 1) aggressive behaviors commonly exhibited in persons with ASPD and AABS may actually push people away from them, 2) the extreme care-seeking behaviors that persons with dependent personality disorder similarly lead others to distance themselves from the person, and 3) persons with avoidant personality disorder are likely to have great difficulty reaching out to others to obtain support. Associations between antisocial syndromes and substance use disorders, bipolar I disorder, ADHD and histrionic personality disorder in persons with PTSD may be a function of difficulties in managing emotions and a propensity for engaging in impulsive and risky behavior. Such tendencies may enhance the likelihood of experiencing traumatic events.

Other reasons for the high levels of co-occurring disorders in persons with antisocial syndromes and PTSD may have to do with diagnostic criteria that are labeled differently but reflect the same underlying process. For example, the mania criterion of 'irritable mood' for 
bipolar I disorder is similar to the 'irritability and aggressiveness' criterion for ASPD. Similarly, the 'suspiciousness' criterion for paranoid personality disorder is similar to the 'hypervigilance' criterion for PTSD. Finally, aggressiveness which characterizes persons with antisocial behavioral syndromes has also been found in persons with obsessive-compulsive personality disorder perhaps explaining the co-occurrence of these disorders.

The authors also examined whether the association between antisocial behavioral syndromes and the high level of other co-occurring disorders may be a reflection of the number of traumatic events experienced. However, statistical tests to determine whether the number of traumatic events is the common link between antisocial behavioral syndromes and high levels of co-occurring disorders did not support that this as a likely explanation for the high level of comorbidity.

Despite the major strength of the study (large sample that is representative of the noninstitutionalized adult US population at large), there are limits to what can be concluded based on the data. First, the study uses a cross-sectional design (data represent a 'snapshot' in time rather than over a period of time). Cross-sectional data only reveals relationships between variables at one point in time, and therefore, we are unable to draw any conclusions about causes of antisocial behavioral syndromes, PTSD, or co-occurring disorders. Another limitation is that the data are self-report and rely on a person's recall of symptom onset and course. Because the sample included only those adults who were non-institutionalized, persons in correctional centers (jails and prisons) were excluded, even though a very high percentage of persons in correctional centers have antisocial behavioral syndromes.

There are several implications of this study for clinical practice and for research. Persons with antisocial behavior and PTSD are likely to have multiple other co-occurring disorders. As 
such, it is essential that clinicians regardless of setting (e.g. outpatient mental health clinic, inpatient mental health unit, addiction treatment, correctional setting) systematically assess for co-occurring disorders rather than limiting their assessment to the presenting problem alone. Also, because persons with ASPD have such poor treatment outcomes, there is an urgent need for research to determine how best to intervene on ASPD at various stage of the life course and to determine whether effective treatments for various psychiatric disorders are also effective in those who also have antisocial behavioral syndromes. Multiple interventions may be requiredeither simultaneously or sequentially--in caring for persons who have multiple other disorders in addition to antisocial behavior and PTSD. Nurses are uniquely positioned to assess and monitor patients with multiple co-occurring conditions 